\title{
Value of preoperative urine white blood cell and nitrite in predicting postoperative infection following percutaneous nephrolithotomy: a meta-analysis
}

\author{
Shuhao Ruan", Zhiyong Chen", Zewu Zhu, Huimin Zeng, Jinbo Chen*, Hequn Chen*^ \\ Department of Urology, Xiangya Hospital, Central South University, Changsha, China \\ Contributions: (I) Conception and design: S Ruan, Z Chen, J Chen, H Chen; (II) Administrative support: J Chen, H Chen; (III) Provision of study \\ materials or patients: S Ruan, Z Chen; (IV) Collection and assembly of data: S Ruan, Z Chen, Z Zhu; (V) Data analysis and interpretation: S Ruan, Z \\ Chen, H Zeng; (VI) Manuscript writing: All authors; (VII) Final approval of manuscript: All authors. \\ \#These authors are joint first authors. \\ *These authors are joint corresponding authors. \\ Correspondence to: Jinbo Chen; Hequn Chen. Department of Urology, Xiangya Hospital, Central South University, Changsha 410008, China. \\ Email: chenjinbo1989@yahoo.com; chenhequnxy@126.com.
}

\begin{abstract}
Background: To evaluate to what degree preoperative urine white blood cell (WBC) and urine nitrite (NIT) values are predictive of postoperative infections following percutaneous nephrolithotomy (PCNL).

Methods: A systematic literature search was performed of the PubMed, Embase, Cochrane Library, Wanfang Data, National Knowledge Infrastructure (CNKI), and China Science and Technology Journal Database (CSTJ or VIP) online databases to identify relevant studies that examined the predictive value of urine WBC or NIT as risk factors for post-PCNL infection, and the search was finished on February 28, 2020. Two independent reviewers screened the relevant studies, extracted necessary data from the eligible case-control studies (CCS), and assessed the quality of included studies through the Newcastle-Ottawa scale (NOS). RevMan 5.3 software and the Stata 16.0 software were used to complete the statistical analysis of data. Results are expressed as odds ratio (OR) with $95 \%$ confidence intervals (CIs).
\end{abstract}

Results: According to the statistical analysis of 12 eligible studies involving 6113 patients, positive urine WBC (WBC+: OR =3.86, 95\% CI: 3.03-4.91, P<0.001) and positive NIT (NIT ${ }^{+}$: OR =7.81, 95\% CI: 5.44$11.21, \mathrm{P}<0.001)$ in preoperative tests were identified as independent risk factors for postoperative infections following PCNL.

Conclusions: In summary, as risk factors for postoperative infections, the presence of preoperative urine $\mathrm{WBC}+$ and NIT+ should be evaluated as part of clinical procedure, in order to reduce infections of PCNL.

Keywords: Percutaneous nephrolithotomy (PCNL); postoperative infections; urine white blood cell (urine WBC); urine nitrite

Submitted May 19, 2020. Accepted for publication Oct 20, 2020.

doi: $10.21037 /$ tau-20-930

View this article at: http://dx.doi.org/10.21037/tau-20-930

\footnotetext{
$\wedge$ ORCID: 0000-0002-4286-9308.
} 


\section{Introduction}

Urinary stones commonly present in urological ward patients (1). Since first being described in 1976 (2), percutaneous nephrolithotomy (PCNL) has been one of the main methods of removing upper urinary tract stones (3). As a minimally invasive surgery involving a small incision, more rapid recovery, and higher stone clearance rate, PCNL has been the first-line treatment for kidney stones $>2 \mathrm{~cm}$ and stones that have a more complex presentation (1). With the development of radiology, ultrasonography, endoscopy, and lithotripsy equipment, the safety and efficacy of PCNL have improved significantly in recent decades. However, surgical complications, especially various infections, remain a cause for concern $(3,4)$.

Fever is one of the common signs of urinary tract infections (UTIs), and may signal the onset of severe UTIs. Systemic inflammatory response syndrome (SIRS) is a common post-PCNL infectious complication that may be confused with urosepsis by clinical medical staff. Urosepsis, identified as the most serious postoperative infectious complication in urological wards, is one type of sepsis related to UTIs (5). Without timely treatment, urosepsis may progress rapidly to uroseptic shock and even multiple organ dysfunction syndrome (MODS), which can be life-threatening (6). Consequently, early prediction and diagnosis of infections is critical in guiding perioperative antibiotic therapy and in reducing serious postoperative infections.

Urine culture is one of several effective measures in the diagnosis of UTIs, but it requires significant time for bacterial culture and may also be compromised by sample contamination. More commonly, clinical urologists prefer to use routine urine tests as a diagnostic tool in their daily practice, because they are less costly and time-consuming. In routine urine tests of patients with urolithiasis, the commonly observed index value includes white blood cell (WBC), red blood cell, and nitrite (NIT) levels. Urinary NIT paired with urine WBC has reportedly been used in diagnosis of UTIs with high accuracy (7). This raises the question of whether or not post-PCNL infections can be predicted by NIT or WBC in preoperative urine tests. Although there have been several relevant individual studies exploring the risk factors of post-PCNL infections, no meta-analysis has been conducted to summarize the existing data. Therefore, we pooled and analyzed current relevant studies to evaluate to what degree preoperative urine WBC and NIT values are predictive of postoperative infections following PCNL.

We present the following article in accordance with the Preferred Reporting Items for Systematic Reviews and Meta-Analyses (PRISMA) reporting checklist (available at http://dx.doi.org/10.21037/tau-20-930) (8).

\section{Methods}

Our study has been registered in International Platform of Registered Systematic Review and Meta-analysis Protocols (registration number INPLASY202060048).

\section{Data sources and search}

Two authors independently searched relevant studies in the PubMed, Embase, Cochrane Library, Wanfang Data, National Knowledge Infrastructure (CNKI), and China Science and Technology Journal Database (CSTJ or VIP) online databases, and the search was finished on February 28, 2020. The search terms were: ("Percutaneous Nephrolithotomy" OR "Percutaneous Nephrolithotripsy" OR "PCNL" OR "MPCNL") AND ("Leukocyte" OR "White Blood Cell" OR "WBC" OR "Nitrite") AND ("Infection" OR "Fever" OR "Sepsis" OR "Urosepsis" OR "Systemic Inflammatory Response Syndrome" OR "SIRS" OR "Shock"). Given the likelihood of potentially relevant studies with high value being included in the reference lists of identified studies, all reference lists were also screened.

\section{Inclusion and exclusion criteria}

Inclusion criteria for the selected relevant studies were the following: (I) the full-text article published in English or Chinese; (II) a case-control study (CCS); (III) data with preoperative urine WBC or NIT.

The exclusion criteria were the following: (I) case reports, conference reports, review articles, and meta-analyses; (II) emergency surgeries; (III) duplicate publications of similar studies from the same author or organization.

\section{Screening of articles}

According to the PRISMA flow diagram (8), the search results from the online databases were imported into the EndNote X9.2 software downloaded from the online library of Central South University. Two independent authors screened the title and abstract of each study and excluded those that were duplicates or irrelevant. The full-text 
articles of the remaining potential studies were downloaded and reviewed carefully. Finally, any disagreement between the two authors was solved by a third independent author or through discussion.

\section{Data extraction and quality assessment}

Relevant data were extracted from the eligible studies by two independent authors, and the consistency between the data was checked by a third independent author. The extracted data included the author names, published year, study types, sample size, race, gender, age, preoperative urine test, and postoperative infectious complications. Two independent authors assessed the quality of the eligible CCS using the Newcastle-Ottawa scale (NOS) (9).

\section{Data synthesis and statistical analysis}

In selected articles, the value of preoperative urine WBC or NIT in predicting postoperative infectious complications was compared in patients undergoing PCNL. The primary outcome data were postoperative sepsis, urosepsis or SIRS, while the secondary outcome data were postoperative fever or septic shock.

In this meta-analysis, the Review Manager software (RevMan Version 5.3, Cochrane Collaboration, Oxford, UK) and the STATA software (Stata version 16.0, Stata Corp LP, College Station, TX, USA), were used to complete the statistical analysis of data. Data type was dichotomous data and the effect measure was odds ratio (OR) with a confidence interval (CI) of $95 \%$ (95\% CI). Mantel-Haenzsel (M-H) was used as the statistical method. The statistical heterogeneity among included studies was tested by the Chi-square test, including the Cochran Q test and $\mathrm{I}^{2}$ test (10). When no significant statistical heterogeneity was detected (both $\mathrm{I}^{2}<50 \%$ and $\mathrm{P}>0.10$ ), the fixed effects (FE) model was used. Otherwise, the random effects (RE) model was used. Because the $\mathrm{I}^{2}$ was unknown initially, the RE model was used provisionally. If significant statistical heterogeneity was present, sensitivity analyses were used to evaluate the reliability of the result, by omitting each study individually.

\section{Results}

Eligible studies, characteristics and quality assessment

According to the search method, 419 potentially relevant studies were identified from the six online databases $($ PubMed $=36$, Embase $=71$, Cochrane $=12$, CNKI $=93$ and Wanfang $=98$, VIP =109). Based on inclusion and exclusion criteria, 207 studies remained after duplicates were removed, a further 18 studies remained after abstract screening, and a final 12 studies remained after full-text review. The selection of studies is shown in Figure 1, and the basic characteristics and bias risk assessment of the 12 eligible studies (11-22), are individually shown in Tables 1,2.

\section{Postoperative infections and urine WBC}

Eleven of the eligible studies reported that preoperative urine WBC positive was an independent risk factor for postoperative infections, while there were different grouping criteria of urine $\mathrm{WBC}$ results, including $\mathrm{WBC}$ $\geq 1+, \mathrm{WBC} \geq 2+$, and $\mathrm{WBC} \geq 3+$.

Five studies (SIRS: Ailaiti W 2019, Fan J 2017, and He Z 2018; Urosepsis: Zhu Z 2020; Septic shock: Wang Y 2012) reported that the ratios of postoperative infections in patients with $\mathrm{WBC} \geq 1+(3.6-20.8 \%)$ were individually higher than WBC $1+(1.6-11.8 \%)$, with a significant difference between the two groups $(\mathrm{OR}=2.98$, 95\% CI: $2.25-3.93, \mathrm{P}<0.001$, Figure 2), with no statistical heterogeneity being detected across the five studies $\left(\mathrm{I}^{2}=16 \%, \mathrm{P}=0.31\right)$.

Three studies (fever: Chen M 2018 and He Y 2016; urosepsis: Chen D 2019) reported that the ratios of postoperative infections in patients with $W B C \geq 2+$ $(5.8-43.2 \%)$ were individually higher than WBC $<2+$ (0.4-12.0\%), with a significant difference between the two groups (OR =6.36, 95\% CI: 3.21-12.59, $\mathrm{P}<0.001$, Figure 2) and no statistical heterogeneity being detected across the three studies $\left(\mathrm{I}^{2}=18 \%, \mathrm{P}=0.29\right)$.

Three studies (urosepsis: Luan G 2018 and Wang X 2018; shock: Cao G 2016) reported that the ratios of postoperative infections in patients with $\mathrm{WBC} \geq 3+$ $(3.8-44.4 \%)$ were individually higher than those of $\mathrm{WBC}<3+(0.3-2.8 \%)$, with a significant difference between the two groups (OR $=15.69,95 \% \mathrm{CI}: 6.87-35.84, \mathrm{P}<0.001$, Figure 2) and no statistical heterogeneity being detected across the three studies $\left(\mathrm{I}^{2}=0 \%, \mathrm{P}=0.68\right)$.

In total, the positive groups were at significantly higher risk of postoperative infections than the negative groups individually $(\mathrm{OR}=3.86,95 \% \mathrm{CI}: 3.03-4.91, \mathrm{P}<0.001$, Figure 2). However, statistical heterogeneity $\left(\mathrm{I}^{2}=62 \%\right.$, $\mathrm{P}=0.004)$ and heavy statistical heterogeneity $\left(\mathrm{I}^{2}=87.9 \%\right.$, $\mathrm{P}=0.0003$ ) were individually detected among the 11 studies 


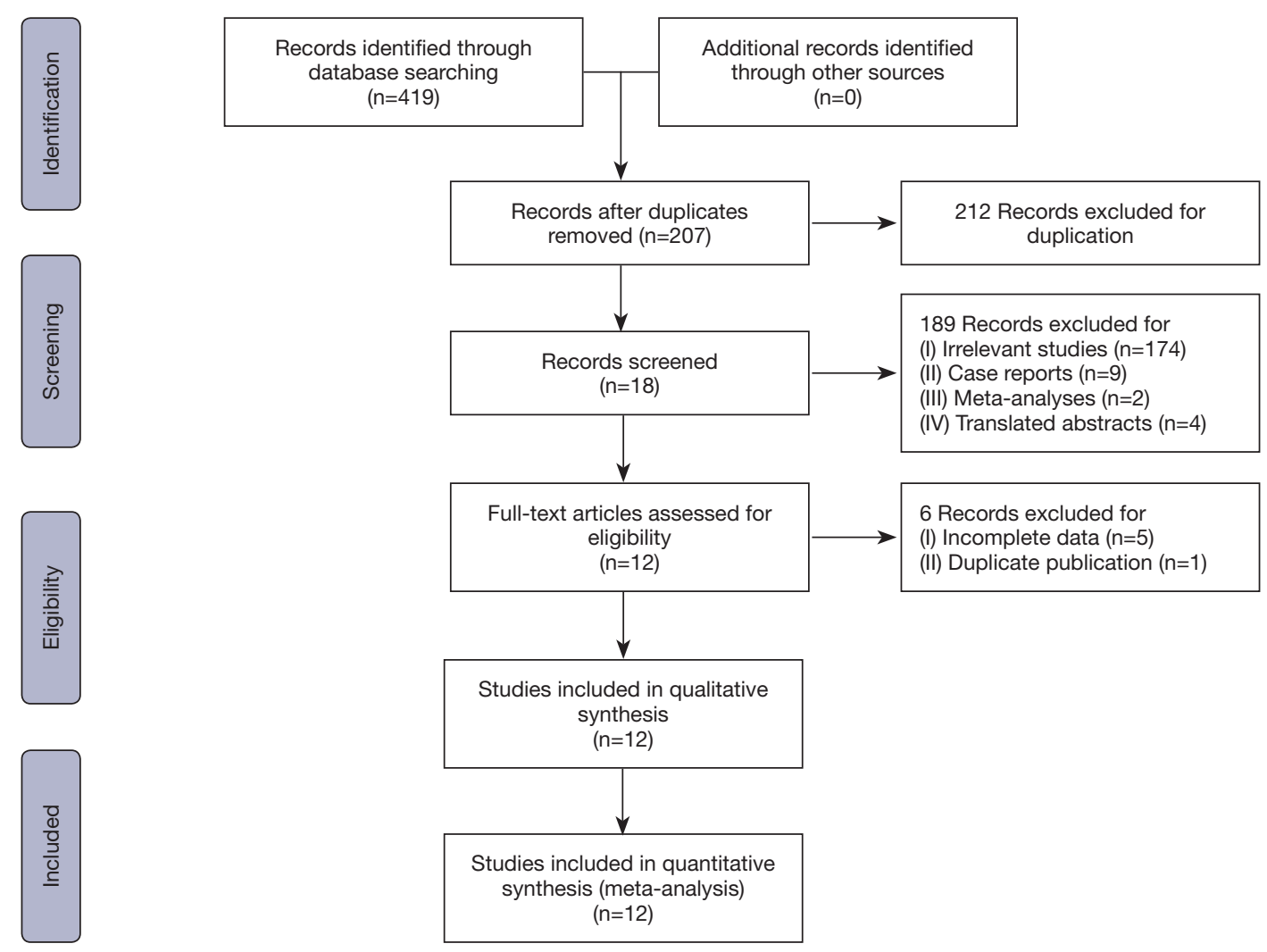

Figure 1 PRISMA flow diagram of study selection. PRISMA, Preferred Reporting Items for Systematic Reviews and Meta-Analyses.

Table 1 Basic characteristics of eligible studies

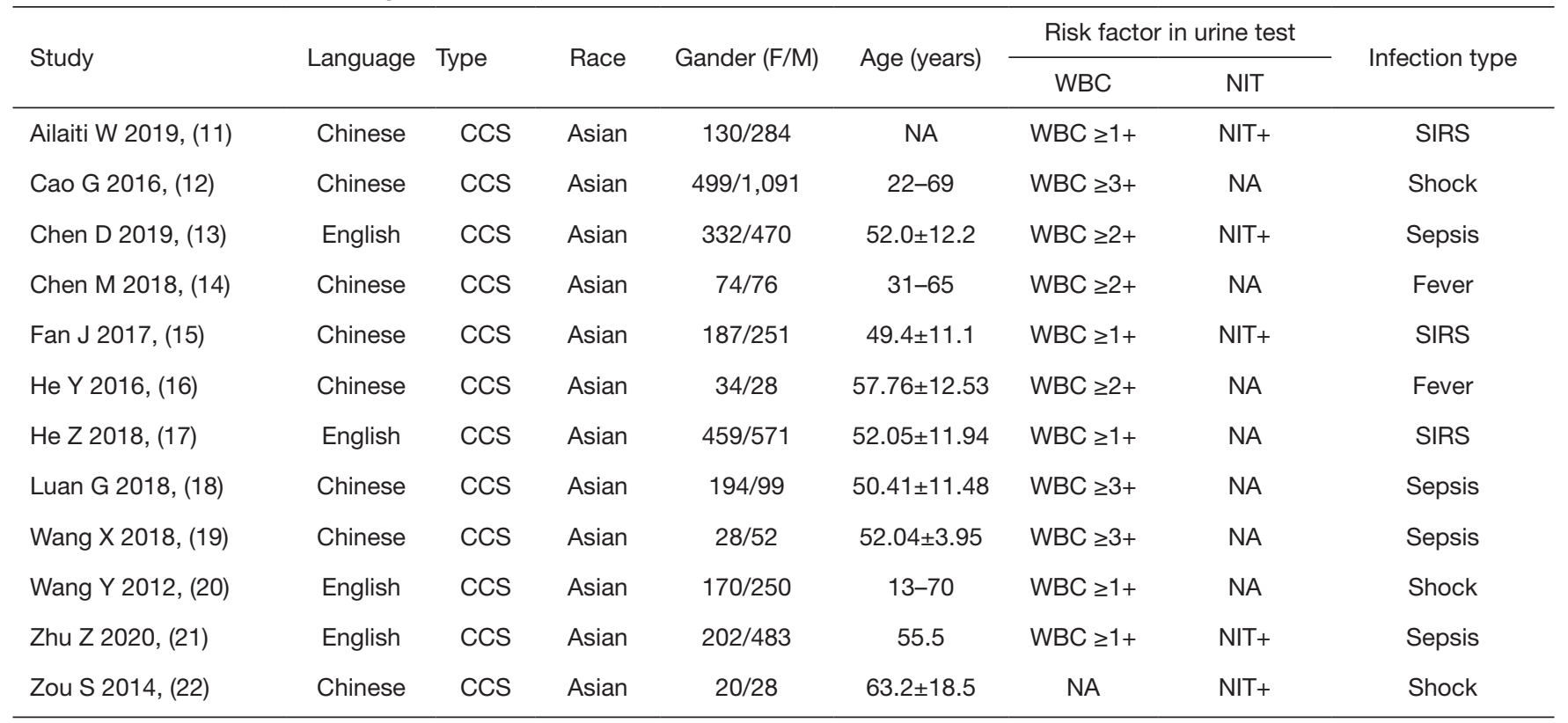

F/M, female/male; SD, standard deviation; NIT+, positive nitrite; WBC+, positive WBC, urine WBC $>25 / \mu \mathrm{L}$ or $>5 / \mathrm{HP}$ (high-power field),

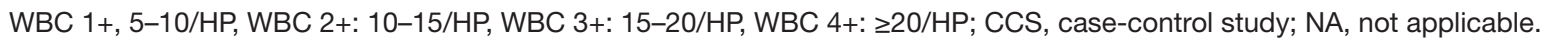


Table 2 Bias risk assessment of eligible studies

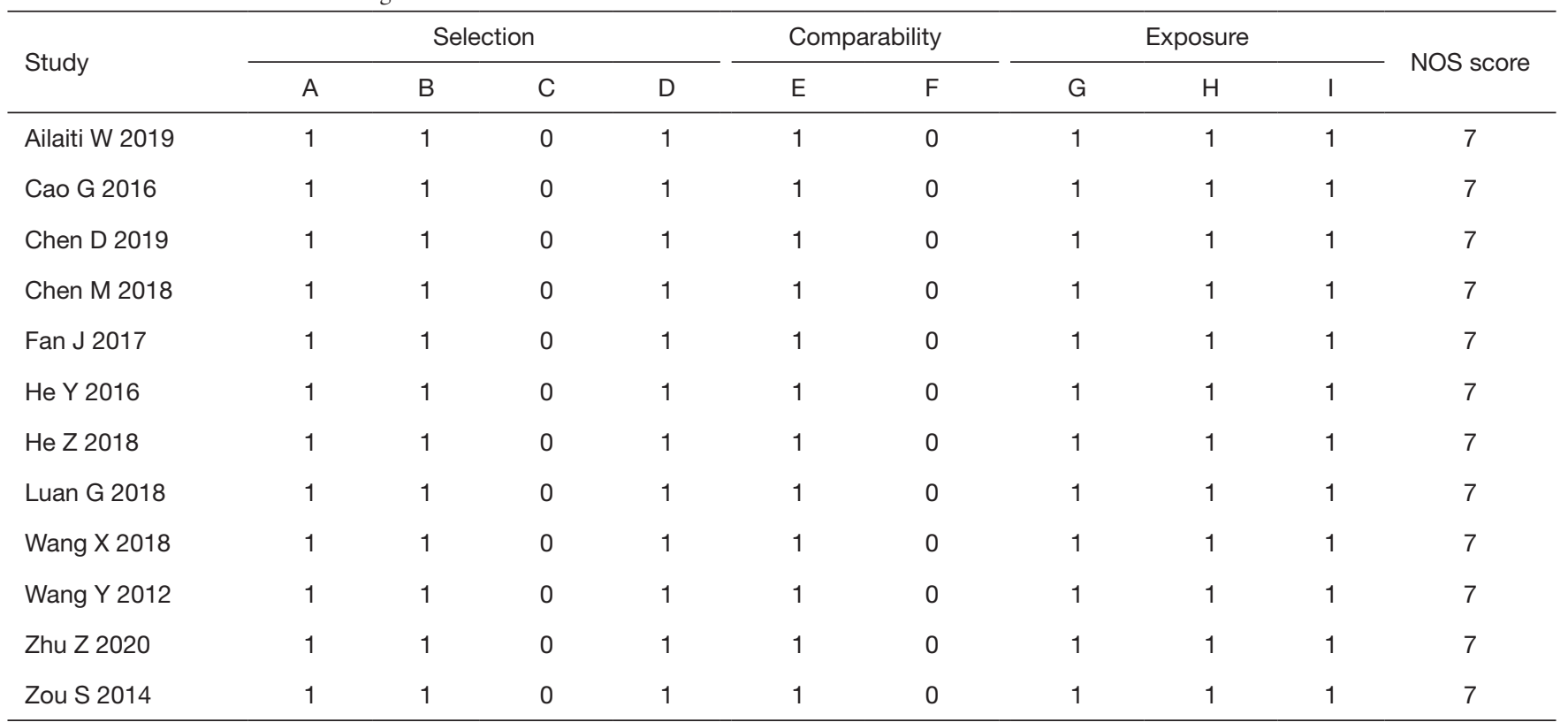

A, adequate case definition; B, representativeness of the cases; C, selection of controls; D, definition of controls; E, study controls for the most important factor; F, study controls for any additional factor; G, ascertainment of exposure; $\mathrm{H}$, same method of ascertainment for cases and controls; I, non-response rate; NOS: Newcastle-Ottawa scale, the NOS score ranges from 0 to 9 , the higher score means the lower risk of bias and the score $\leq 5$ means a high risk of bias.

and the 3 subgroups, both of which were mainly caused by the different grouping criteria of different subgroups.

\section{Postoperative infections and urine NIT}

Five eligible studies reported that preoperative urine NIT positive was an independent risk factor for postoperative infections (SIRS: Ailaiti W 2019 and Fan J 2017; urosepsis: Chen D 2019 and Zhu Z 2020; septic shock: Zou S 2014). The ratios of postoperative infections in the positive groups $(8.7-43.8 \%)$ were individually higher than the ratios in the negative groups (0.9-9.9\%), with a significant difference between the two groups $(\mathrm{OR}=7.81,95 \% \mathrm{CI}$ : 5.44-11.21, $\mathrm{P}<0.001$, Figure 3), and no significant statistical heterogeneity being detected across the five studies $\left(\mathrm{I}^{2}=0 \%\right.$, $\mathrm{P}=0.43)$.

\section{Postoperative infections and urine WBC \& NIT}

Additionally, Chen et al. reported that combined WBC+ and NIT+ in preoperative urine tests was considered an early and rapid predictor of post-PCNL urosepsis ( $\mathrm{OR}=3.9,95 \% \mathrm{CI}: 1.2-12.1, \mathrm{P}=0.021$ ), and the incidence of postoperative infections (fever, SIRS and urosepsis) in the group of patients with combined WBC+ and NIT+ was significantly higher than that in the groups of patients with WBC- and NIT-, WBC+ and NIT-, or WBC- and NIT+ $(\mathrm{P}<0.01)(13)$. Zhu et al. also reported that positive NIT, $\mathrm{WBC}$, and leukocyte esterase (LE) (NIT+, WBC+, and $\mathrm{LE}+$ ) in preoperative urine tests was an independent risk factor for urinary sepsis (OR $=17.51,95 \%$ CI: 6.75-45.38, $\mathrm{P}<0.001)(21)$.

\section{Publication bias and sensitivity analyses}

Through the inverted funnel plot visual inspection, no significant publication bias was found in the present study (Figure 4). Additionally, the sensitivity analyses were performed by individually omitting every eligible study, and the statistical robustness tests in the two comparisons were both successful (Figure 5).

\section{Discussion}

Although there has been considerable progress achieved in PCNL since 1976, postoperative infectious complications following PCNL continue to be problematic for urologists. Therefore, it is necessary to identify risk factors for post- 


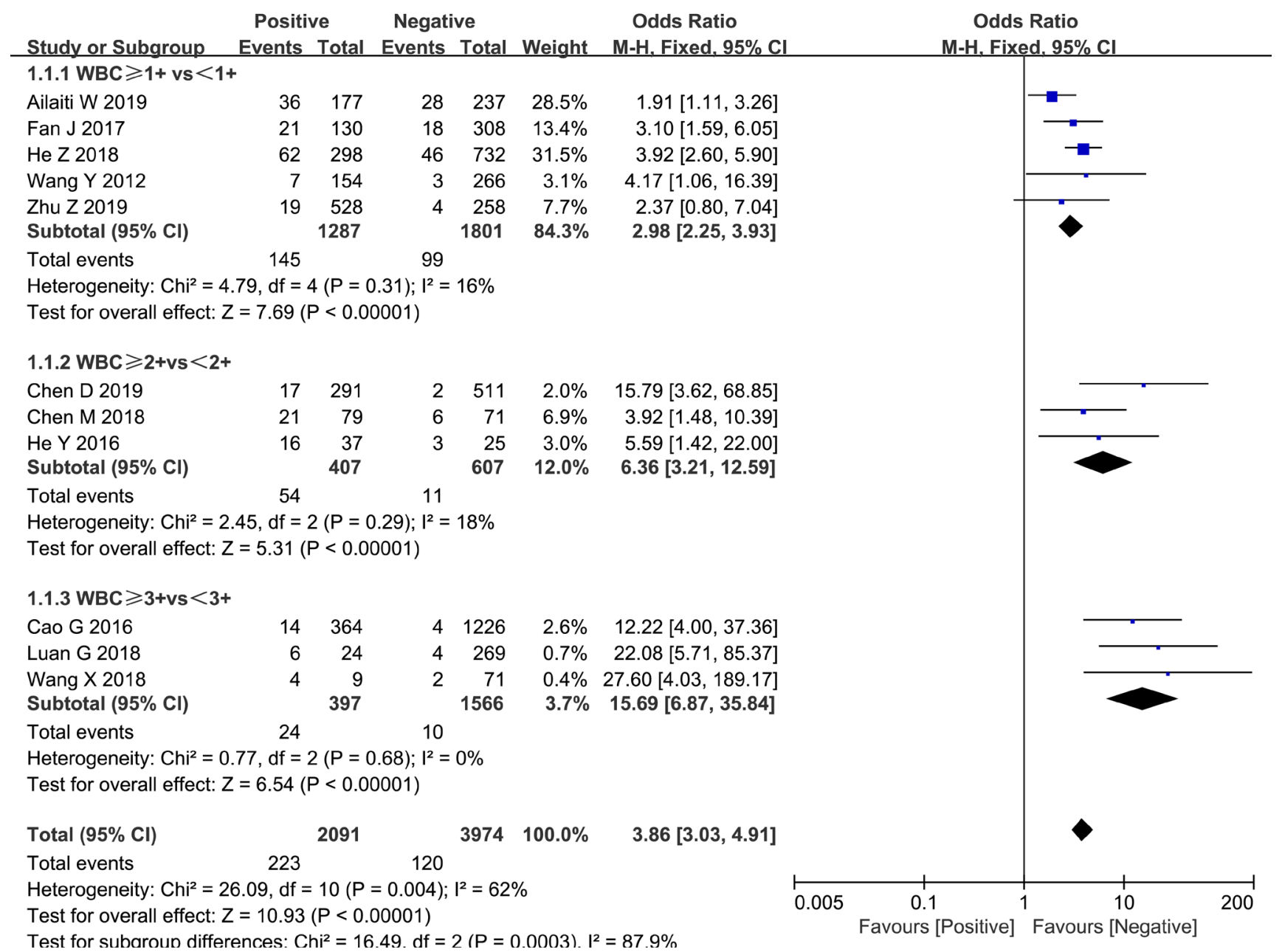

Figure 2 Postoperative infections and urine WBC. WBC, white blood cell; M-H, Mantel-Haenzsel; CI, confidence interval.

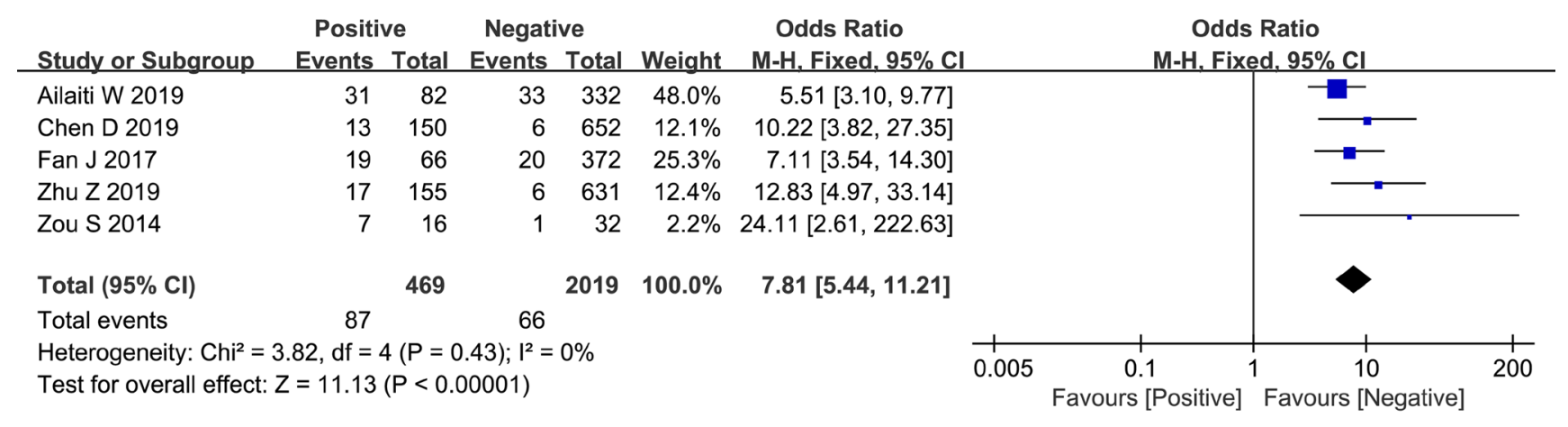

Figure 3 Postoperative infections and urine NIT. NIT, nitrite; M-H, Mantel-Haenzsel; CI, confidence interval. 

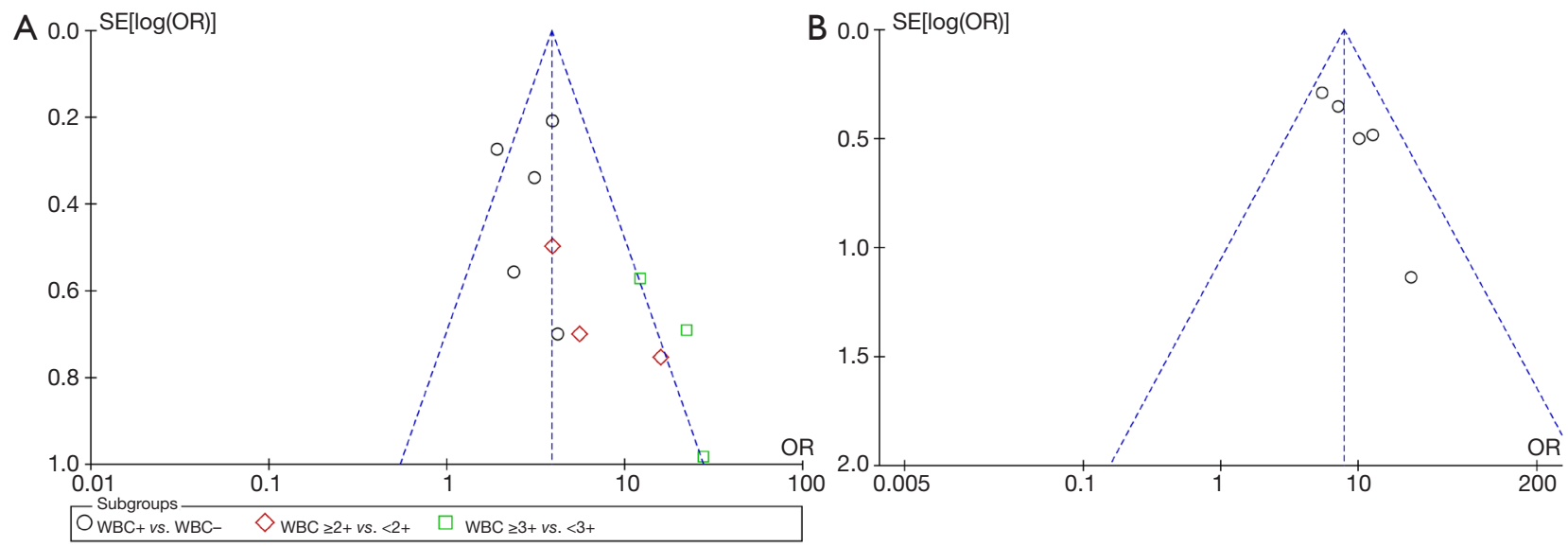

Figure 4 Funnel plot of comparison regarding (A) WBC and (B) NIT. WBC, white blood cell; NIT, nitrite; SE, standard error; OR, odds ratio.
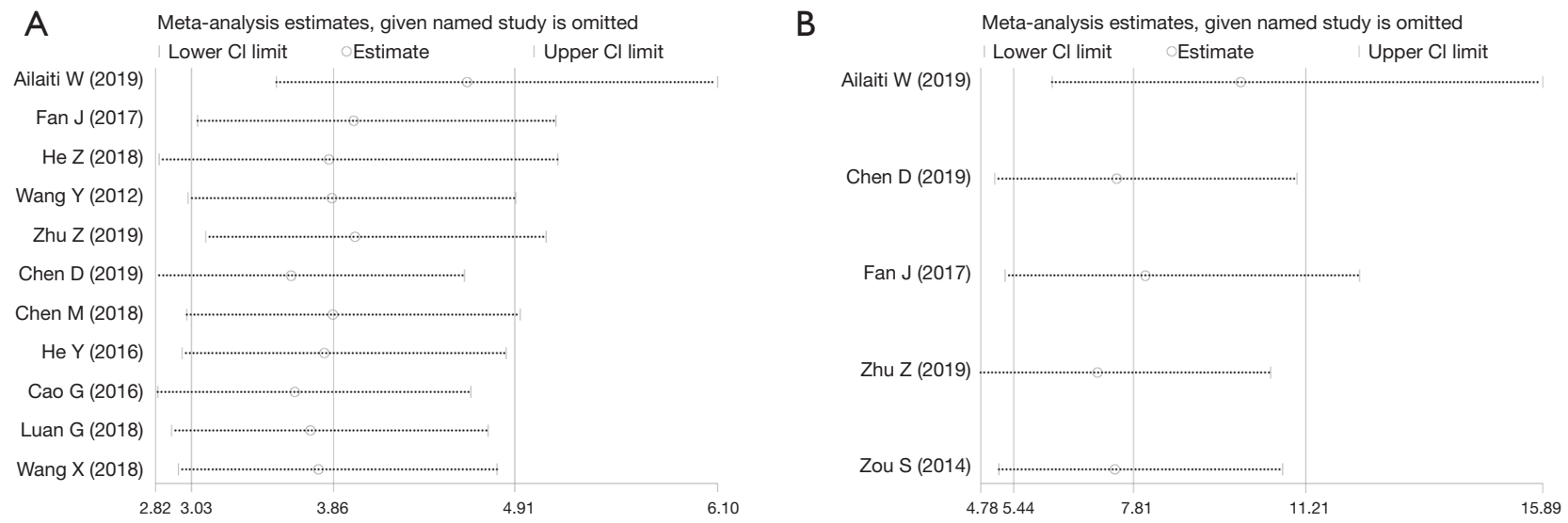

Figure 5 Sensitivity analysis of comparison regarding (A) WBC and (B) NIT. WBC, white blood cell; NIT, nitrite; CI, confidence interval.

PCNL infections in order to predict the likelihood of postoperative infections. Our meta-analysis conclusively indicated that positive WBC and NIT in preoperative urine tests were two independent risk factors for postoperative infectious complications in patients undergoing PCNL.

Of the eligible studies, five compared $\mathrm{WBC}+$ with $\mathrm{WBC}-(\mathrm{OR}=2.98, \mathrm{P}<0.001)$, three compared $\mathrm{WBC} \geq 2+$ with $\mathrm{WBC}<2+(\mathrm{OR}=6.36, \mathrm{P}<0.001)$, and three compared WBC $\geq 3+$ with $W B C<3+(\mathrm{OR}=15.69, \mathrm{P}<0.001)$. Patients with positive urine $\mathrm{WBC}$ had a significantly higher risk of postoperative infections. For instance, Wang et al. reported that the ratio of septic shock in patients with urine WBC $\geq 25 / \mu \mathrm{L}$ was four times higher than that of patients with $\mathrm{WBC}<25 / \mu \mathrm{L}(20)$; furthermore, Chen et al. reported that the ratio of urosepsis in patients with urine $\mathrm{WBC} \geq 10 / \mathrm{HP}$ was 14.9 times higher than that in patients with $W B C<10 /$ HP (13). Consistently, WBC counting in urine sediment microscopy and NIT in urine tests could be used in the diagnosis of UTIs (7). Additionally, recurrent UTI was reported as an independent risk factor for both post-PCNL SIRS (OR $=2.08,95 \% \mathrm{CI}: 1.03-4.20, \mathrm{P}=0.04)$ and sepsis $(\mathrm{OR}=23.71,95 \%$ CI: 3.75-150.04, $\mathrm{P}=0.01)$ (5).

Five studies compared NIT + with NIT $-(\mathrm{OR}=7.81$, $\mathrm{P}<0.001)$. The ratios of postoperative SIRS in patients with NIT+, were 3.8-5.4 times higher than those of NIT0032 $(11,15)$, and the ratios of postoperative urosepsis in patients with NIT+, were 9.4-11.5 times higher than those of NIT$(13,21)$. Additionally, in another relevant study of the same 
first author, Fan et al. reported that positive urine NIT was also an independent risk factor in uroseptic shock in patients with post-PCNL SIRS (OR $=10.570, \mathrm{P}=0.025)$ (23). Bacteria in the urine tract can convert nitrate to NIT, and then the NIT dipstick can test this rapidly and measure bacteriuria indirectly, which can be used in the diagnosis of UTIs with high specificity (95\%) but low sensitivity (51\%) (24). Furthermore, the presence of urine LE, tested by the LE dipstick, could significantly increase the diagnostic accuracy of UTIs. The sensitivity and specificity of urine dipstick (nitrate and LE in combination) was $22.9 \%$ and $99.1 \%$ individually (24). It was also reported that urine NIT paired with WBC could significantly increase the diagnostic accuracy of UTIs, and that the disjunctive pairing of NIT and WBC in patients whose bladder incubation time $\geq 4$ hours had higher efficacy (84\%) than the NIT $(81 \%)$ and the WBC (72\%), with high specificity (95\%) but low sensitivity (41\%) (7). The advantage of a urine test of WBC and NIT is that it takes only a few hours, whereas urine culture and drug sensitive tests need several days, which may delay diagnosis and treatment. Consequently, urine tests of WBC and NIT should be regarded as the method of choice for predicting postoperative infections on urological wards.

There were several limitations in the present study. First, only 12 studies conducted in hospitals of different levels in China met our inclusion criteria, which might have created a risk of bias. Second, there were differences in postoperative infectious complications among the included studies, which included fever, SIRS, sepsis, and shock. Third, there were other confounding risk factors that cannot be ignored including female sex, older age, higher body mass index (BMI), persistent urinary obstruction, severe hydronephrosis, higher stone burden, infectious stones, longer operation time, and antibiotic therapy $(5,13,20,21,25)$. Therefore, more case control studies with larger sample sizes and detailed data may be needed to optimize the reliability of the present study.

\section{Conclusions}

Both NIT+ and WBC+ in preoperative urine tests have been found to be independent risk factors for postoperative infectious complications of patients undergoing PCNL. Accordingly, to reduce the occurrence of severe postoperative infections, it is suggested that urologists pay close attention to PCNL patients with preoperative urine $\mathrm{WBC}+$ or NIT+ values, and especially combined WBC+ and NIT+ values.

\section{Acknowledgments}

We would like to thank all authors of each included study. Funding: This study was supported by the National Natural Science Foundation of China (no. 81770705 to Hequn Chen).

\section{Footnote}

Reporting Checklist: The authors have completed the PRISMA reporting checklist. Available at http://dx.doi. org/10.21037/tau-20-930

Conflicts of Interest: All authors have completed the ICMJE uniform disclosure form (available at http://dx.doi. org/10.21037/tau-20-930). The authors have no conflicts of interest to declare.

Ethical Statement: The authors are accountable for all aspects of the work in ensuring that questions related to the accuracy or integrity of any part of the work are appropriately investigated and resolved. Ethical approval was not required as relevant data were extracted without compromising individual privacy.

Open Access Statement: This is an Open Access article distributed in accordance with the Creative Commons Attribution-NonCommercial-NoDerivs 4.0 International License (CC BY-NC-ND 4.0), which permits the noncommercial replication and distribution of the article with the strict proviso that no changes or edits are made and the original work is properly cited (including links to both the formal publication through the relevant DOI and the license). See: https://creativecommons.org/licenses/by-nc-nd/4.0/.

\section{References}

1. Turk C, Petrik A, Sarica K, et al. EAU Guidelines on Interventional Treatment for Urolithiasis. Eur Urol 2016;69:475-82.

2. Fernstrom I, Johansson B. Percutaneous pyelolithotomy. A new extraction technique. Scand J Urol Nephrol 1976;10:257-9.

3. Cai C, Liu Y, Zhong W, et al. The Clinical Application of New Generation Super-Mini Percutaneous Nephrolithotomy in the Treatment of $\geq 20 \mathrm{~mm}$ Renal 
Stones. J Endourol 2019;33:634-8.

4. Kyriazis I, Panagopoulos V, Kallidonis P, et al. Complications in percutaneous nephrolithotomy. World J Urol 2015;33:1069-77.

5. Koras O, Bozkurt IH, Yonguc T, et al. Risk factors for postoperative infectious complications following percutaneous nephrolithotomy: a prospective clinical study. Urolithiasis 2015;43:55-60.

6. Whitehurst L, Jones P, Somani BK. Mortality from kidney stone disease (KSD) as reported in the literature over the last two decades: a systematic review. World J Urol 2019;37:759-76.

7. Ferry SA, S EH, Ferry BM, et al. High Diagnostic Accuracy of Nitrite Test Paired with Urine Sediment can Reduce Unnecessary Antibiotic Therapy. Open Microbiol J 2015;9:150-9.

8. Moher D, Liberati A, Tetzlaff J, et al. Preferred reporting items for systematic reviews and meta-analyses: the PRISMA statement. BMJ 2009;339:b2535.

9. Stang A. Critical evaluation of the Newcastle-Ottawa scale for the assessment of the quality of nonrandomized studies in meta-analyses. Eur J Epidemiol 2010;25:603-5.

10. Higgins JP, Thompson SG, Deeks JJ, et al. Measuring inconsistency in meta-analyses. BMJ 2003;327:557-60.

11. Ailaiti W, Hamulati T, Anniwaer Y. Risk factors of systemic inflammatory response syndrome after percutaneous nephrolithotomy and establishment of prediction models. Journal of Minimally Invasive Urology 2019;8:40-5.

12. Cao G, Qiu X, Li Z, et al. Risk factors of septic shock after mini-percutaneous nephrolithotripsy. China Journal of Endoscopy 2016;22:10-3.

13. Chen D, Jiang C, Liang X, et al. Early and rapid prediction of postoperative infections following percutaneous nephrolithotomy in patients with complex kidney stones. BJU Int 2019;123:1041-7.

14. Chen M, Huang D, Xu M, et al. Analysis of correlative factors of fever after percutaneous nephrolithotomy. China Journal of Endoscopy 2018;24:12-6.

15. Fan J, Wu W, Zhu W, et al. Systemic inflammatory response syndrome after percutaneous nephrolithotomy:

Cite this article as: Ruan S, Chen Z, Zhu Z, Zeng H, Chen J, Chen H. Value of preoperative urine white blood cell and nitrite in predicting postoperative infection following percutaneous nephrolithotomy: a meta-analysis. Transl Androl Urol 2021;10(1):195-203. doi: 10.21037/tau-20-930 an assessment of risk factors. Chinese Journal of Urology 2017;38:857-61.

16. He Y, Su X, Lin T, et al. The Influencing Factors and Countermeasures of Complications of Kidney Stones Treated by B Ultrasound-Guided Percu-taneous Nephrolithotripsy. Clinical Medical \& Engineering 2016;23:857-8.

17. He Z, Tang F, Lei H, et al. Risk factors for systemic inflammatory response syndrome after percutaneous nephrolithotomy. Prog Urol 2018;28:582-7.

18. Luan G, Wang Q, Qian B, et al. Risk factors of urinary sepsis after percutaneous nephrolithotripsy. Journal of Bingtuan Medicine 2018;(1):27-30.

19. Wang X. Risk factors of urinary sepsis after percutaneous pneumatic nephrolithotomy for renal stones. Modern Diagnosis \& Treatment 2018;29:3865-6.

20. Wang Y, Jiang F, Wang Y, et al. Post-percutaneous nephrolithotomy septic shock and severe hemorrhage: a study of risk factors. Urol Int 2012;88:307-10.

21. Zhu Z, Cui Y, Zeng H, et al. The evaluation of early predictive factors for urosepsis in patients with negative preoperative urine culture following mini-percutaneous nephrolithotomy. World J Urol 2020;38:2629-36.

22. Zou S, Deng R. The analysis of nitrite between shock and urinary infection after percutaneous nephrolithotomy. Medicine and Health Care 2014;22:20,22.

23. Fan J, Wan S, Liu L, et al. Predictors for uroseptic shock in patients who undergo minimally invasive percutaneous nephrolithotomy. Urolithiasis 2017;45:573-8.

24. Demilie T, Beyene G, Melaku S, et al. Diagnostic accuracy of rapid urine dipstick test to predict urinary tract infection among pregnant women in Felege Hiwot Referral Hospital, Bahir Dar, North West Ethiopia. BMC Res Notes 2014;7:481.

25. Liu C, Zhang X, Liu Y, et al. Prevention and treatment of septic shock following mini-percutaneous nephrolithotomy: a single-center retrospective study of 834 cases. World J Urol 2013;31:1593-7.

(English Language Editors: D. Fitzgerald and J. Gray) 